\title{
Calculation of Average Characteristics of Water Environment Stratification
}

\author{
A.E. Pogrebnoy \\ Marine Hydrophysical Institute, Russian Academy of Sciences, Sevastopol, \\ Russian Federation \\ e-mail: pogrebok57@mail.ru
}

\begin{abstract}
Problems arising at applying traditional procedure for averaging hydrophysical characteristics' vertical profiles are analyzed. The conclusion on necessity of taking into account vertical displacements of fluid elements is drawn. For this purpose, the Lagrangian invariants, (particularly, the averaged background distribution of the density field) are proposed to be applied. It permits to calculate the averaged depths of the vertical structure various elements. If the fluid is stratified the surfaces of constant density is called isopycnal. They nowhere intersect and divide the fluid into the layers. In the ideal fluid (when the processes of diffusion/exchange are absent), its elements never cross the isopycnal surfaces. Note that in real ocean the exchange processes are always present. As a result the fluid element density can change; thereupon it leaves its isopycnal level. Therefore the in situ density is not the true Lagrangian invariant. The average value for each element corresponding to the average depth of this element is used as an average vertical distribution of a sought parameter. The means for increasing reliability of the stratification elements' identification and applicability of the proposed method are discussed. The present method developed based on the thermodynamics general principles can also be applied for averaging the atmosphere parameters and the laboratory experiments. Besides, use of density as the Lagrangian invariant is not obligatory. The basic advantage of such a choice is conditioned by its monotonous dependence on depth. As well as for the ocean, in case there are uniformities of the parameter vertical gradient which permits to identify the stratification elements, additional background averaging can be used. The averaging vertical scale has to exceed the characteristic scales of small heterogeneities (for example, high-gradient streaks), but it is to be smaller than the background heterogeneities' scales (mixed layers' thicknesses). In case the denoted gradient heterogeneities are absent additional averaging is not required.
\end{abstract}

Keywords: substantial derivative, Lagrangian invariant, stepped structure.

DOI: 10.22449/1573-160X-2015-3-72-77

(C) 2015, A.E. Pogrebnoy

(C) 2015, Physical Oceanography

Introduction. Averaging of the hydrophysical values' vertical profiles is one of the most common procedures in the experimental hydrophysics. Horizontal spatial averaging of the vertical stratification parameters is necessary for describing the basins' statistical characteristics and for the ocean geographical zoning. Temporal averaging is done to describe diurnal, synoptic, seasonal, inter-annual and other types of variability. Average climatic distributions of density profiles are also used in the ocean numerical models $[1,2]$ for calculating variability of the velocity field baroclinic component.

Another important application of the averaging procedure is data interpolation: averaging of two or more adjacent vertical profiles (with the required weighting coefficients) provides the intermediate interpolated distribution of the parameter under study. Such a frequent use of the profiles' averaging procedure allows its attributing to standard ones.

As for the stratification average characteristics, it should be borne in mind that the data averaging procedure requires limitation of the data sample by a set of profiles which include all the basic structural elements. For example, the profiles' samples intended for describing characteristics of the seasonal thermocline should not contain simultaneously the data of summer and winter (when the thermocline is ruined) probings. 
Drawbacks of the traditional averaging method. The procedure of averaging the hydrophysical parameters' vertical distribution is traditionally represents the following. For each selected depth horizon, the average value of the studied parameter is found. Just such a procedure is used in one of the most popular packages for numerical processing of hydrophysical data GrADS [3].

However, a profound analysis shows that the characteristic structural features inherent to each individual profile can be significantly distorted or even completely absent in the resultant average profile obtained by the traditional method.

The essence of the foresaid is shown in Fig. 1. Averaging of two profiles with a stepped distribution of any parameter (for example, seasonal thermocline) can lead to appearance of two steps (instead of one) in the resultant average profile. Hence, at averaging of a large number of such profiles, quantity of structural features can increase or, vise versa, the features inherent to each individual profile can completely vanish.
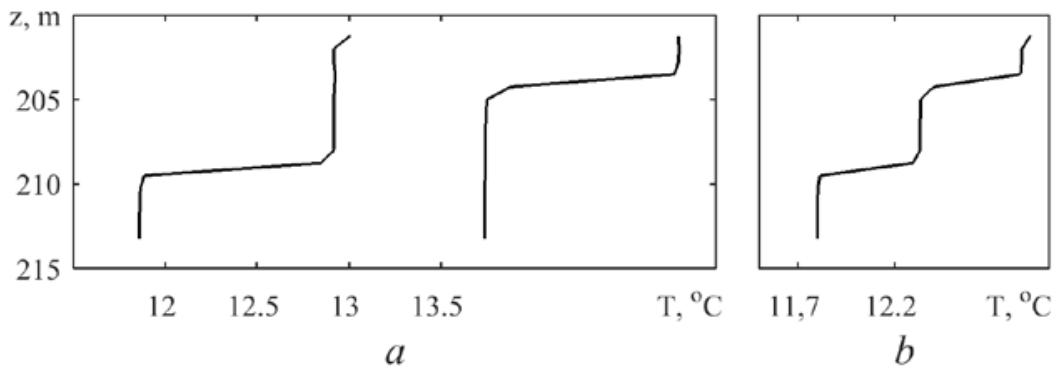

Fig. 1. Averaging of two temperature profiles: $a$ - fragments of individual profiles ( $x$-axis scaling corresponds to temperature, ${ }^{\circ} \mathrm{C}$, for the left profile, the temperature values for the right profile are increased by $\left.2^{\circ} \mathrm{C}\right) ; b$ - the result of the represented profiles' averaging by the traditional method (averaging is done along the $x$-axis)

The phenomenon of the structural features' splitting and smearing is similar to that resulted from a stroboscopic shooting of moving objects. As a rule, the number of objects on the resultant image is equal to the number of stroboscopic flashes, or the individual features of a moving object are completely smeared.

In hydrophysics vertical motion of the fluid elements can be conditioned by the internal waves' effect. Spatial variability of the structural features' depth can also be related to horizontal gradients of the background structure (for example, near the frontal zones).

This effect is mathematically connected with the fact that variability of any hydrophysical parameter $\lambda$ is described by equation [4]

$$
\frac{d \lambda}{d t} \equiv \frac{\partial \lambda}{\partial t}+(\mathbf{u} \nabla) \lambda=F+D
$$

where $\mathrm{u}(t, \mathrm{x})=\dot{\mathrm{x}}(t)$ is the fluid motion velocity in the point $\mathbf{x}$ at the time moment $t$, term $\frac{\partial \lambda}{\partial t}$ describes explicit dependence on time, and $(\mathbf{u} \nabla) \lambda$ is the effect of the parameter $\lambda$ spatial variability (in the along-flow direction) upon its temporal variability, $F$ is the function of a source/drain, $D$ is the summand related to diffusion. At that the operator

$$
\frac{d}{d t} \equiv \frac{\partial}{\partial t}+(\mathrm{u} \nabla)
$$


is called a substantial or individual derivative. If we confine ourselves to considering the movements only along the vertical coordinate $z$ the operator of the individual derivative is written in the following form

$$
\frac{d}{d t} \equiv \frac{\partial}{\partial t}+w \frac{\partial}{\partial z},
$$

where $w$ is the vertical velocity.

The main difference of operators (2) and (3) from the temporal derivative in the solid case is presence of the latter term in equations (2) and (3) which reflects variation of the fluid elements' location in space. It is just this term stipulates occurring of the effect of the structural features' splitting/smearing when the traditional in hydrophysics method of averaging the parameters' spatial distributions is applied.

The proposed method of averaging. Based on the foresaid, the proposed method of averaging should not only take into account explicit dependence of the parameter under examination upon time, but also provide consideration of possible vertical displacements of the fluid structural elements. The defined goal can be achieved by step-by-step solving of the following tasks: profiles;

- identification of the fluid (structure) components of the individual averaged

- calculation of the averaged depths of these elements;

- calculation (for each element) of the average value of the parameter under study; at that it is assumed to correspond to the calculated average depth of this element.

To identify the elements of the fluid column vertical structure, it is desirable to use any scalar parameter of the medium the value of which absolutely identifies the fluid element. For this purpose the substantial derivative in equation (1) has to be necessarily equal to zero. Such scalar parameters are called in hydrophysics the Lagrangian invariants. Their values remain the same along the fluid motion trajectory.

The basic feature of the Lagrangian invariant follows from its definition - it is passively transported by a moving fluid. In other words, its value remains constant for each individual fluid particle, and only its location changes.

For a potential vortex, in [5, 6] density and potential temperature were used for the stratified ocean and the atmosphere, respectively, as such an invariant in deducing the Rossby - Ertel theorem.

In case of a stratified fluid, the surfaces of constant density are called isopycnal. They nowhere intersect and divide the fluid into the layers. In an ideal fluid (in the absence of diffusion/exchange), its elements never cross the isopycnal surfaces.

Note that in real ocean the exchange processes are always present. As a result, the fluid element density can change, and owing to this fact it leaves its isopycnal level. That is why the in situ density is not a true Lagrangian invariant.

Let us analyze what can be done to increase reliability of the stratification elements' identification. For this purpose, consider equation (1) for density. Due to the evaporation/precipitation and heating/cooling processes, the source/drain function $F$ can be different from zero on the sea surface and on the bottom. In the stratified areas the main water column, just the nonzero diffusion term $D$ leads to 
violation of the Lagrangian effect. For its approximation in a one-dimensional case, the following relation is most often used:

$$
D=\frac{\partial}{\partial z}\left(\alpha \frac{\partial \rho}{\partial z}\right)
$$

where $\alpha$ the exchange coefficient. Note that representation of the diffusion term in this form is acceptable for the majority of exchange processes: molecular diffusion, turbulence, double diffusion and others.

It follows from (4) that the extreme values of $D$ correspond to the areas with abrupt changes of the density gradient or the exchange coefficient. In natural conditions, the areas with the increased values of $\alpha$ are localized in the very place where variability of the vertical gradient is observed. For example, the maximum shear of the surface currents' velocity that can induce instability is concentrated near the seasonal thermocline. Therefore, the case of spatial variability of the exchange coefficient will not be specifically considered.

Note that the diffusion influence upon the identification parameter invariance is conditioned by spatial variability of its gradient which can be significantly reduced by preliminary smoothing. Therefore the density background (vertically averaged) field is proposed to be used (instead of the in situ density) as an identifier of the structure elements.

While selecting the vertical scale of smoothing it is desirable to be guided by the following: averaging should result in maximum reduction of abrupt gradient drops; at that it is necessary to preserve the general background structure of the density field.

In the ocean, there are two classes of structures with well-pronounced abrupt drops of the density gradient: the structures of the stepped and intrusion types. The intrusions (in contrast to the stepped structures) are characterized by nonmonotonic temperature/salinity vertical distribution. The density fields in both cases are qualitatively identical. They are practically the homogeneous layers (their thickness is a few tens or even hundreds meters) separated by the high-gradient streaks with a few meters thickness.

For the described structures, vertical scale of the density field background averaging should be higher than the high-gradient streaks' thickness and lower than the characteristic scale of the mixed layers. If the density field structure is characterized by absence of the pronounced features and its vertical gradient is uniform, the diffusion term is small, choice of the averaging scale is not decisive and the averaging itself loses its meaning.

It is known that heterogeneities in the stratified ocean are stretched mostly horizontally. Their horizontal scale is by a few orders larger than the vertical one that results in formation of the elements of the so-called fine structure. In the case, when it comes to horizontal spatial averaging of the vertical stratification characteristics, let us consider the structure unchangeable in time and dependent only on horizontal and vertical coordinates.

In case of horizontal averaging and in order to identify the vertical stratification elements, the background density value averaged over the abovementioned vertical scale (as well as in case of time averaging) is proposed to be used as the Lagrangian invariant. The additional advantage of this choice is expressed in both cases in the fact that the fluid density is, as usual, a monotone function of depth. Consequently, there exists a one-to-one relation between depth $z$ and density $\rho$ which suggests the inverse function $Z(\rho)$. 
Using individual $Z(\rho)$ for each profile one can calculate the averaged depths $\langle Z\rangle$ of various fluid elements of the vertical structure. The example of such a calculation is given in Fig. 2. One should pay attention to qualitative difference between the averaging results shown in Fig. $1, b$ and $2, b$.
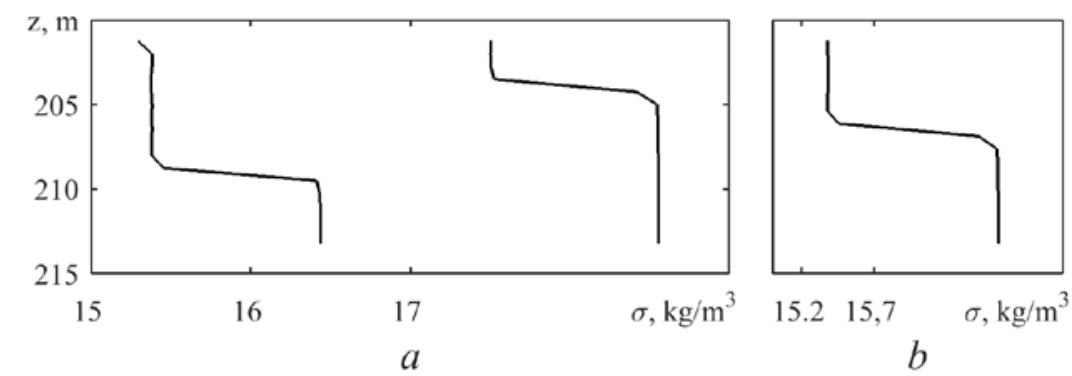

Fig. 2. Averaging of the stratification elements' depths for two density profiles: $a$ - fragments of the density individual profiles ( $x$-axis scaling corresponds to the density standard units (stand. unit) for the left profile, density values for the right profile are increased by 2 stand. units); $b$ - profile of average values of the isopycns' depths (averaging is carried out along the $y$-axis)

Finally, the average (isopycnal) value for each fluid element corresponding to the average depth of this element will be used as an average vertical distribution of this (sought) parameter.

Example of application the proposed method. The benefits of the proposed method as compared to the traditional approach are demonstrated in Fig. 3. The initial data are represented by a fragment of the section carried out in the northwestern Tropical Atlantic between the points with coordinates $10.030{ }^{\circ} \mathrm{N}$, $49.875^{\circ} \mathrm{W}$ and $10.355^{\circ} \mathrm{N}, 50.758{ }^{\circ} \mathrm{W}$. Fine structure of the temperature and salinity fields in this region is characterized by a stepped stratification of the main pycnocline which is observed over thousands of kilometers and conditioned by convection of the salt fingers $[7,8]$.
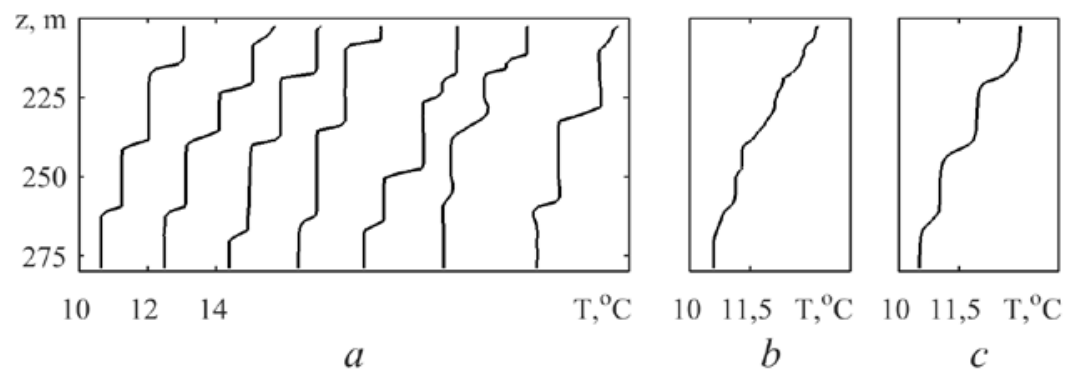

Fig. 3. Averaging of a series of temperature profiles measured between the points with coordinates $10.030^{\circ} \mathrm{N}, 49.875^{\circ} \mathrm{W}$ and $10.355^{\circ} \mathrm{N}, 50.758^{\circ} \mathrm{W}: a$ - fragments of individual profiles $(x$-axis scaling corresponds to the temperature, ${ }^{\circ} \mathrm{C}$, for the first profile; the temperature values for each subsequent profile are increased by $2{ }^{\circ} \mathrm{C}$ ); $b$ - the result of averaging the represented profiles by the traditional method; $c$ - result of averaging by the method proposed in the paper

Fig. 3, $a, b, c$ shows the individual temperature profiles, as well as the ones averaged by the traditional method and by the proposed technique. It is noteworthy that the middle profile constructed traditionally shows practically no structural features (steps) of the individual profiles. Whereas on the profile constructed by the 
proposed method they are present. Note that the results of the salinity profiles' processing are similar.

Thus, having preserved the initial structure, the average vertical distributions of hydrophysical parameters contain information on the average depth ranges of fine-structure features and average heat- and salt-content of homogeneous layers. The traditional approach did not permit to achieve such results.

Experience in application of this technique shows that in order to obtain the background structure, the vertical scale $10 \mathrm{~m}$ is usually sufficient and even in the Gulf Stream frontal zone it does not exceed $20 \mathrm{~m}$. The scale of the data represented in Fig. 3 was $4.5 \mathrm{~m}$. Its twofold increase (just for this set of probes) does not result in any quantitative changes. The criterion of the averaging scale selection for obtaining background distribution of the density field is described above.

Conclusions. This method developed based on general principles of thermodynamics is also applicable to averaging the atmospheric parameters and to laboratory experiments. Besides, application of density as the Lagrangian invariant is not obligatory. The basic advantage of such a choice is conditioned by its monotonous dependence on depth. As well as for the ocean, the additional background averaging can be used in case of presence of inhomogeneities of the parameter vertical gradient which permits to identify the stratification elements. The averaging vertical scale should be larger than the typical dimensions of fine inhomogeneities (for example, high-gradient streaks), but smaller than the scales of the background inhomogeneities (thickness of the mixed layers). If the indicated gradient inhomogeneities are absent the additional averaging is not required.

\section{REFERENCES}

1. Blumberg, A.F., Mellor, G.L., 1987, “A description of a three-dimensional ocean circulation model [Three-Dimensional Coastal Ocean Models]”, Ed N.S. Heaps. Washington, DC, American Geophysical Union, 208 p.

2. Mellor, G.L., 1998, "User’s Guide for a Three-dimensional, Primitive Equation, Numerical Ocean Model Version 1998”, Princeton, Princeton University, 41 p.

3. Doty, B., 1995, "The Grid Analysis and Display System. GrADS V1.5.1.12", http://www.doty@cola.iges.org.

4. Landau, L.D., Lifshits, E.M., 1987, "Fluid Mechanics [Vol. 6 (2nd ed.) of A Course of Theoretical Physics]”, Pergamon Press, 539 p.

5. Rossby C.-G., 1940, "Planetary flow patterns in the atmosphere", Quart. J. Roy. Meteorol. Soc., vol. 66, pp. 68-87.

6. Haynes, P.H., McIntyre, M.E., 1987, "On the evolution of vorticity and potential vorticity in the presence of diabatic heating and frictional or other forces”, J. Atmos. Sci., vol. 44(4), no. 1 , pp. 828-840.

7. Ohotnikov, I.N., Pogrebnoi, A.E., 1993, “Tonkaja vertikalnaja struktura na termohalinnom fronte $v$ oblasti stupenchatogo rassloenija [Thin vertical structure on the thermohaline front in the area of stepped stratification]”, Izvestiya, Atmospheric and Oceanic Physics, vol. 28, no. 12, pp. 1218-1222.

8. Panteleev, N.A., Pogrebnoi, A.E., 1993, “O raschete vertikalnih potokov plavuchesti, obuslovlenih konvektsiei solevih paltsev [On calculation of the buoyancy vertical fluxes conditioned by convection of salt fingers]”, Okeanologia, vol. 33, no. 4, pp. 501-506. 